\title{
Could the Level in Parasitaemia of Plasmodium Determine Sensitivity to Various Diagnostic Tests?
}

\author{
Obed Nanjul Goselle ${ }^{1 *}$, Godwin Yandu Ajiji', Anvou Jambol'2, Joseph Terhema Sunday ${ }^{3}$, \\ Ojochemi Sunday Idoko', Shedrach Sunday Udoh', Oliseemeka Charles Ejete1, \\ Yahaya Mbaya Ahmadu', Henrietta Oluwatoyin Awobode', Godwin Nyiutaha Imandeh", \\ Bernard Malau Matur ${ }^{1}$
}

\author{
${ }^{1}$ Applied Entomology and Parasitology Unit, Department of Zoology, University of Jos, Jos, Nigeria \\ ${ }^{2}$ National Veterinary Research Institute, Vom, Nigeria \\ ${ }^{3}$ Health and Development Support Programme, Jos, Nigeria \\ ${ }^{4}$ Department of Zoology, University of Ibadan, Ibadan, Nigeria \\ ${ }^{5}$ Department of Zoology, Federal University of Agriculture, Makurdi, Nigeria \\ Email: *obeto247@yahoo.com
}

How to cite this paper: Goselle, O.N., Ajiji, G.Y., Jambol, A., Sunday, J.T., Idoko, O.S., Udoh, S.S., Ejete, O.C., Ahmadu, Y.M., Awobode, H.O., Imandeh, G.N. and Matur, B.M. (2020) Could the Level in Parasitaemia of Plasmodium Determine Sensitivity to Various Diagnostic Tests? American Journal of Molecular Biology, 10, 224-245. https://doi.org/10.4236/ajmb.2020.103015

Received: May 12, 2020

Accepted: July 13, 2020

Published: July 16, 2020

Copyright $\odot 2020$ by author(s) and Scientific Research Publishing Inc. This work is licensed under the Creative Commons Attribution International License (CC BY 4.0).

http://creativecommons.org/licenses/by/4.0/ (c) (i) Open Access

\begin{abstract}
The discovery of Plasmodium parasites and its incrimination as the principal cause of malaria in humans has continued to excite researchers towards inventing possible easier methods of diagnosing and identifying these pathological agents in order to mitigate, control and eliminate its continuous scourge to humanity. Currently, three diagnostic methods have been proposed, but agreements as to whether the level of parasitaemia in an individual could connote likely confirmations in the three methods i.e. gold standard, RDTs' and PCR/NESTED PCR, have continued to be a subject of debate. To lay to rest the debate as reported in many studies, we collected blood samples from 100 symptomatic patients who reported to the Jos-Nigeria hospital and using the gold standard methods, we were able to confirm that $30(30 \%)$ samples out of the 100 blood samples collected were positive to $P$. falciparum, chiefly recorded among duffy-negative Africans. Excited with our findings, we prepared the thick blood films for each sample and used it to estimate the levels of parasitaemia (parasites density) per $\mu$ of blood (i.e. $1+; 2+; 3+$ and $4+$ ) per 100 high power fields (|HPF). We then subjected the individually confirmed parasite density samples to the other two methods i.e. Rapid Diagnostic Test (one-step RTD and optimal-IT ${ }^{\oplus} \mathrm{RDT}$ ) and to molecular assay (PCR and the nested PCR). Interestingly, of the 30 positive samples, 18 (60\%) were confirmed positive to the one-step and optimal-IT ${ }^{\circledast}$ RDTS, while $3(30 \%)$ out of the $10(100 \%)$ samples of various parasite density subjected to molecular as-
\end{abstract}


say (PCR and the nested PCR) were positive to only P. falciparum. Statistical analysis of variance based on single factor computed using SPSS indicates a no significant difference $(\mathrm{P}>0.05)$ in the parasitaemia levels of the four groups/categories of patients; i.e. variance ratio of 0.011976 calculated was less than F-critical (2.816466) at 5\% (0.05). Whereas gold standard could be considered as the optimal method, for the PCR/NESTED PCR, the sensitivity is dependent on high level of parasitaemia.

\section{Keywords}

Plasmodium, Parasitaemia, Diagnostic Tests, Malaria, Sensitivity

\section{Introduction}

With half of the world population ( 3.2 billion) at risk, 214 million cases recorded each year and an estimated 438,000 deaths, human malaria caused by traditionally five species of Plasmodium has continued to be a serious public health concern. Sub-Saharan African continues to carry a disproportionately high share of the burden as it is home to $89 \%$ of malaria cases and $91 \%$ of malaria deaths. Particularly, Plasmodium falciparum is reported to be the dominant species in Sub-Saharan African, with its origin still a highly debated topic [1] [2] [3].

Although global reports indicate the decline in the scourge of the disease [4], previous reports indicate that despite the Nigerian government efforts in domesticating and flagging off of the Roll Back Malaria Project, Nigeria still suffers the world's greatest burden, with approximately 51 million cases and 207,000 deaths reported annually (approximately $30 \%$ of the total malaria burden in Africa), while $97 \%$ of the total population (approximately 173 million) is at risk of infection. Consequently, culminating into malaria accounting for $60 \%$ of outpatient visits to hospitals; approximately $11 \%$ maternal mortality and $30 \%$ child mortality, especially among children less than 5 years [5] [6].

Effective control and management of malaria always require presumptive, quick, sensitive, accurate and cost-effective diagnostic methods. The presumptive diagnosis involved the use of signs and symptoms, whereas quick, sensitive, accurate and cost-effective diagnosis had always involved demonstration of the parasite, its parts, products in body fluids and the drug efficacy and resistance profile based on recommended treatments [6] [7].

Despite the evolution of malaria parasites diagnosis, the traditional microscopic examination of stained thin and thick blood films otherwise termed the "gold standard" has always been recommended [8]. Notwithstanding, various problems which include time-consuming laboratory procedures, considerable training requirement, expertise in microscopic examination, well-maintained microscope, well-stained slides [9], errors in estimation and quantification for low-density parasitaemia (10 to 100 parasites/ $\mu$ of blood) and higher parasitaemia densities of $>5000 / \mu \mathrm{l}$ and especially $>20,000 / \mu \mathrm{l}$ of blood [10], leading to prob- 
lems in the output of epidemiological studies in resource poor settings [11] [12] [13] have been recorded. Thus, new effective and sensitive technologies were developed and introduced to overcome these limitations.

In the last few Years, Rapid Diagnostic Tests (RDTs) or Immuno-chromatograhic test (lateral flow immune-chromatographic antigen detection tests) which does not require a laboratory, electricity or any special equipment had been developed and recognized as an ideal method for diagnosing several infectious diseases, including malaria [9] [14] [15] [16] [17] [18]. On the calculations for parasitaemia below (pg 5); we could modify by including: The number of parasites counted/the number of leucocytes counted ( 200 or 500$) \times 8000$ and the resultant complex is captured on the strip by a band of bound antibody, forming a visible line (T-test line) in the results window with a control line (c-control line) giving information on the integrity of the antibody-dye conjugate, but does not (unless specified) confirm the ability to detect parasite antigen [3] [19] [20]. Although designed to bring significant advantages in malaria control and management with a clear plan of outcomes i.e. drug treatment and appropriate further investigations [16] [21], wide variations in the sensitivity of RDTs [14] [22], and false-negative results might be generated in testing samples with low level parasitaemia [14]. Thus, WHO recommended parasitologist who are using RDTs for malaria diagnosis, to use in conjunction with other methods to confirm the results, characterize infection, and monitor treatment [1].

Consequently, some problems noticed in the output of epidemiological studies e.t.c. could be overcome using molecular techniques such as PCR, which has proved more sensitive and specific than microscopic evaluation and can help to detect infection in patients with low level of parasites and mixed infections at the peripheral level and thus improving the management of infectious diseases, especially in resource-limited settings [23]-[28]. Furthermore, molecular characterization of parasites can be used for any survey on drug resistance, pathogenicity, and molecular epidemiology. Many studies have been done in recent years in other countries to detect malaria using the nested PCR and microscopy [29] [30].

In this study, in order to lay to rest the continuous debate as to the appropriate method to use that could holistically confirm the level of parasitaemia and specificity of parasites, three methods of microscopic examination (gold standard), Rapid Diagnostic Tests, and Polymerase Chain Reaction (PCR) and nested PCR were comparatively evaluated. The aim of this study was to assess and compare between the accuracy of the traditional microscopic examination with the RDTs and PCR methods. Other objectives included: comparing the LDH-based RDT (Optimal) and One Step Malaria Antigen Rapid Test Bio Line with the traditional microscopic examination of stained thin and thick blood films; comparing the traditional microscopic examination of stained thin and thick blood films with nested PCR methods; comparing the LDH-based RDT (Optimal) and One Step Malaria Antigen Rapid Test Bio Line with the nested PCR and to evaluate the data in comparison with previous reports in other stu- 
dies/countries.

\section{Materials and Methods}

\subsection{Study Area}

The study was carried out in Jos North L.G.A of Plateau State, North central Nigeria. Its geographical coordinates are $9^{\circ} 55^{\prime} 0^{\prime \prime}$ North, $8^{\circ} 54^{\prime} 0^{\prime \prime}$ East. It stretches for approximately $104 \mathrm{~km}$ from North to South, $80 \mathrm{~km}$ from East to West covering an area of about $8600 \mathrm{~km}^{2}$. The altitude ranges from around 1200 meters (about $4000 \mathrm{ft}$ ) to a peak of 1829 meters above sea level in Shere Hill (Figure 1). The climate is similar to that of temperate, but situated in the tropics. It is located at a higher altitude which gives Jos-Plateau a near temperate climate with an average temperature of between $18^{\circ} \mathrm{C}$ and $22^{\circ} \mathrm{C}$. The coldest weather and harmattan winds are experienced between December and February. The dry season months of March and April have the warmest temperature. The mean annual rainfall varies from $131.75 \mathrm{~cm}$ in Southern part to $146 \mathrm{~cm}$ on the Plateau. The highest rainfall is recorded during the wet season months of July and August [31].

\subsection{Ethical Consideration}

Ethical approval/clearance to carry out the research work was obtained from the ethical committee of the Plateau specialist Hospital Jos, Plateau State, before the collection of blood samples.

\subsection{Blood Samples Collection}

Collection of blood samples was done at random from patients, who presented clinical signs and symptoms of malaria, and were prescribed for malaria test by the physicians. The collection was done through vein puncture technique as described by Epidi et al. [32]. According to the method, the puncture site was swabbed with a cotton wool dipped in methylated spirit (methanol), followed by a puncture made using a new sterile $2 \mathrm{ml}$ syringe. The blood collected was then transferred into a sterile EDTA (Ethylene Diamine Tetra acetic Acid) container. Each sample was labeled appropriately with patient's name and date of collection.

\subsubsection{Preparation of the Thick Blood Films}

Thick Blood Films were used for parasite identification and estimation of parasite density. A drop of blood from each of the samples collected was placed on a clean grease-free slide. Using the corner of a clean slide, the drop of the blood was spread in a circle the size of a dime (Diameter $1-2 \mathrm{~cm}$ ). The slides were allowed to air-dry thoroughly. The slides were dipped briefly in water to haemolyse (de-haemoglobinize) the red blood cells. Caution was taken in not making too thick films and/or allowing it to dry thoroughly, so as to prevent the films from falling off when staining. 10\% Giemsa stain was prepared and used to stain the smear on the slides for 10 minutes. The slides were rinsed using clean water and allowed for 5 minutes. The slides were then kept uprightly in rack and allowed to air-dry [33]. 


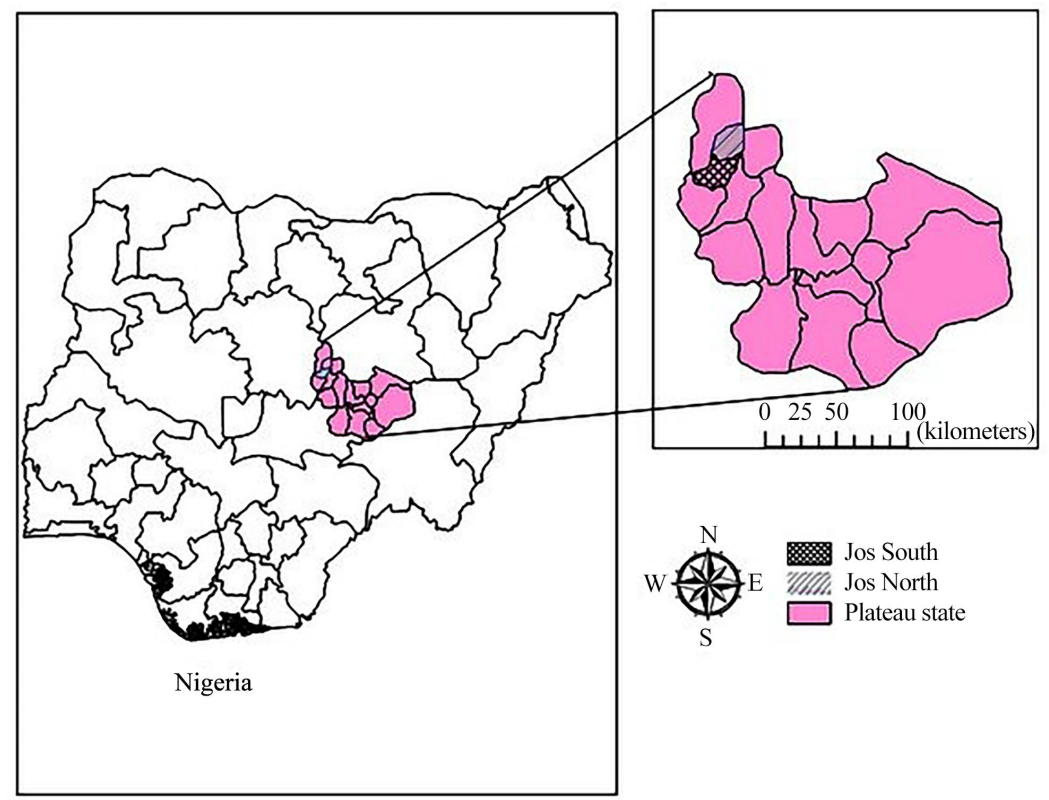

Figure 1. Map of plateau state showing Jos North local government (Plateau State Ministry of Lands and Survey).

\subsubsection{Parasite Density (Parasite per Micro Liter ( $\mu$ l) of Blood)}

This was based on the number of parasites per $\mu$ of blood in a thick blood film, assessed in relation to a predetermined number of leukocytes. An average of 8000 leukocytes per $\mu \mathrm{l}$ was taken as the standard. Before counting began, the equivalent of $0.25 \mu \mathrm{l}$ of blood (100 fields, using a $\times 7$ ocular and a $\times 100$ oil immersion objective) was examined in each thick film to determine the parasite species and stages that might be present. When the parasites had been found, parasite density was calculated using the following counting procedure: Parasites and leukocytes were counted separately using two tally counters. If after 200 leukocytes had been counted, 100 or more asexual parasites had been identified, the results were recorded, showing parasites per 200 leukocytes. If after 200 leukocytes had been counted, 99 or less asexual parasites had been counted, the counting continued until 500 leukocytes had been counted and the results recorded as parasites per 500 leukocytes. In each case, the parasite count in relation to the leukocyte count was converted to parasites per $\mu$ of blood by the simple formula:

Parasite density $($ parasite per $\mu \mathrm{l})=\frac{\text { The number of parasites counted }}{\text { The number of leukocytes counted }} \times 8000$

This meant that if 200 leukocytes were counted, the parasites were multiplied by 40 , and if 500 leukocytes were counted, the parasites were multiplied by 16 . The "plus system" was used to record the levels of parasitaemia (parasite density) as follows: $1+=1-10$ parasites per 100 oil-immersion thick smear field; $2+$ $=11-100$ parasites per 100 oil-immersion thick smear field; $3+=1-10$ parasites per thick smear field; $4+=>10$ parasites per thick smear field (Kumar, YouTube Video). 


\subsubsection{Preparation of Thin Blood Films}

Thin Blood Films are useful in detecting malaria parasite species based on morphology. A drop of blood from each sample was placed at one end of a clean grease-free slide using a Pasteur pipette. A clean spreader slide was held at $54^{\circ}$ angle toward the drop of the blood and allowed to spread along the edge of the spreader slide. The spreader slide was pushed gently forward drawing the blood until the whole slide was smeared. The smears on the slides were allowed to dry completely. The slides were fixed briefly in methanol (100\% or absolute) for 15 30 seconds and allowed to dry completely. The slides were placed on staining rack and $10 \%$ Giemsa stain was applied over it for 10 minutes. This was then rinsed with water. The underneath of the slides were cleansed with cotton wool and placed on a rack to air-dry [34].

\subsubsection{Identification of Human Plasmodium Species}

A drop of oil immersion was placed on the thin and thick stained blood films before they were examined under the microscope using the $\times 100$ objective lens. A careful examination of several microscopic fields of the slide was carried out to detect and identify Plasmodium species presents. The "basic malaria microscopy" [35] was used as a guide in identifying the morphological features of the blood stages that characterize the human Plasmodium species.

\subsection{Rapid Diagnostic Tests (RDTs)}

The blood samples used for the microscopic examination of human Plasmodium species were also used for the RDTs examination. The patient's blood was mixed with a lysing agent in a test strip or well. The lysing agent ruptures the red blood cells, releasing more protein of the parasites. The dye-labeled antibody, specific for target antigen was the control line or band (C) in a plastic well provided with strip and the antibody, also specific for the target antigen, was bound to the strip in a thin test line $(\mathrm{T})$. Blood and buffer, which were placed in their respective wells, mixed with labeled antibody and were drawn up the strip across the lines of bound antibody. The presence of antigen (parasite protein) resulted or lead to the trapping and accumulation of some labeled antibody antigen complex on the test line $(\mathrm{T})$. The excess labeled antibody was trapped and accumulated on the control line (C). The visible control line (C) indicated that labeled antibody had traversed the full length of the strip, past the test line, and that at least some free antibody remained conjugated to the dye and that some of the capturing properties of the antibodies remained intact. The intensity (thickness) of the test line or band varies with the parasite density (antigen concentration) i.e. the higher the parasite density, the thicker the band and vice versa [2].

\subsubsection{Optimal-IT ${ }^{\circledR}$}

The optimal-IT ${ }^{\otimes}$ tests were performed, according to manufacturer's instruction similar to the RDTs procedure outlined. The optimal-IT ${ }^{\circledR}$ was an immunochromatographic test, using monoclonal antibodies (Mabs) against the metabolic en- 
zyme PLDH (Parasite Lactate Dehydrogenase) of Plasmodium (Parasite antigen). These Mabs were classified in two groups; one specific for Plasmodium falciparum and the other was a pan-specific Mab, which reacted with all four species of human Plasmodium; $P$. falciparum, $P$. vivax, $P$. ovale, and $P$. malariae. The interpretation of the assay test strip results was done as; when one control band and two test bands appeared, the test was considered to be positive for Plasmodium falciparum; when one control band and one test band appeared, the test was considered positive for Plasmodium vivax, when only one control band appeared at the top of the test strip without test band, the test was considered to be negative [36].

\subsubsection{One Step RDT}

Some RDTs can detect only one species (Plasmodium falciparum) while others detect multiple species ( $P$. vivax, $P$. malariae, and $P$. ovale). Blood for the test is commonly obtained from a finger prick [2] [37].

\subsection{Molecular Assay}

\subsubsection{Design of Primers}

The primers used for the PCR were synthesized at the inqaba biotechnical limited Hatfield-Pretoria, South Africa. The primer pairs and their sequences are given below: rPLU5 ( $5^{1}$-CCTGTTGTTGCCTTAAACTTC- $3^{1}$ ) and rPLU6 (5 ${ }^{1}$ TTAAAATTGTTGCAGTTA AAACG- $3^{1}$ ) for initial implication reaction of targeted sequence for all species of Plasmodium [38] [39]. For specific detection of Plasmodium species, the primers pair as designed by Incardona et al. [39] were used i.e. $\mathrm{rFAL} 1$ ( $5^{1}$-TTA AAC TGG TTT GGG AAA ACC AAA TAT ATT-3 ${ }^{1}$ ) and rFAL2 ( $5^{1}$-ACA CAA TGA ACT CAA TCA TGA CTA CCC GTC- $\left.3^{1}\right)$ for $P$. falciparum detection; rVIV1 (5 ${ }^{1}$-CGC TTC TAG CTT AAT CCA CAT AAC TGA TAC- $3^{1}$ ) and rVIV2 ( $5^{1}$-AAG GAA AGA AAG TCC TTA- $3^{1}$ ) for $P$. vivax detection. These primer pairs were obtained and stored at about $-20^{\circ} \mathrm{C}$ in the Applied Molecular Biology Laboratory of the Applied Biotechnology Division at the National Veterinary Research Institute Vom, Nigeria. Dilution or reconstitution of the primers were made and stored under the same condition prior to usage.

\subsubsection{Primers Dilution and Recombination}

The materials needed for PCR Primer Dilution (re-suspension) are: molecular grade water, primers (dry), and sterile micro centrifuge tube. Primers are often transported and received in a lyophilized state. First create a master $100 \times$ stock (for each primer and then dilute it to a 10× working stock) as described by FGRS [40] and Stupar Lab [41]. This reduces the number of freeze/thaw cycles that the master primer stock goes through and reduces the chances of contaminating the primary source for the primer.

\subsubsection{Deoxyribonucleic Acid (DNA) Extraction Procedure}

$100 \mu \mathrm{l}$ of the blood sample was taken from each EDTA bottle containing whole 
blood using a $100 \mu \mathrm{l}$ micropipette and dispensed into $1.5 \mathrm{ml}$ centrifuge tubes. $400 \mu$ (i.e. $4 \times$ the volume of the blood (4:1)) of genomic lysis buffer was added to each of the $1.5 \mathrm{ml}$ centrifuge tubes. The mixtures in the $1.5 \mathrm{ml}$ centrifuge tubes were vortexed (Mixed) for 4 - 6 seconds and then let to stand at room temperature for 5 - 10 minutes. The vortexing was done using a vortexing machine. The mixtures from each tube were transferred to a spin column in a collection tube. This was then centrifuged for one minute at $12,000 \mathrm{rpm}$ in a centrifuge. The spin column was transferred into a new collection tube. $200 \mu \mathrm{l}$ of DNA Pre-wash buffer was added to the spin column. This was then centrifuged for 1 minute at $12,000 \mathrm{rpm} .500 \mu \mathrm{l}$ of g-DNA wash buffer was added to the spin column. This was also centrifuged for 1 minute at 12,000 rpm. Each of the spin column was transferred to a clean microcentrifuge tube. $>50 \mu \mathrm{l}$ DNA Elution buffer was added to the spin column and incubated for $2-5$ minutes at room temperature, and then centrifuged for 30 seconds to elute (filter) the DNA. The eluted DNA can be used immediately for molecular-based applications or stored at $<-20^{\circ} \mathrm{C}$ for future use [42].

\subsubsection{Polymerase Chain Reaction (PCR) Protocol}

Three pairs of primers (6 oligonucleotides) which include: rPLU5 and rPLU6, rFAL1 and rFAL2, and rVIV1 and rVIV2 were used. In an initial amplification reaction, the primer pair rPLU5 and rPLU6 was used. The size of DNA target, amplified by these outer primers, was about $1200 \mathrm{bp}$. These primers were genus specific and can, therefore, amplify the target sequences from all four species of human malaria parasite (Plasmodium falciparum, $P$. vivax, $P$. malariae, and $P$. ovale).

The second reaction of Nested PCR (this reduces the amount of non-specific binding because in the second reaction, most of the amplicons of the first reaction only contain the target sequence and its surrounding sequences [43]) was performed for the specific detection of the above Plasmodium species using a set of two primer pairs; rFAL1 and rFAL2 for $P$. falciparum, and rVIV1 and rVIV2 for $P$. vivax detection. Each $20 \mu \mathrm{l}$ reaction mixture for the first amplifications contained $5 \mu \mathrm{l}$ of template DNA, $2 \mu \mathrm{l}$ of $10 \times$ PCR buffer $(50 \mathrm{mM} \mathrm{KCl}, 10 \mathrm{mM}$ Tris- $\mathrm{HCl}$ ), $4 \mathrm{mM} \mathrm{MgCl} 2,200 \mu \mathrm{M}$ of each dNTP's, 0.4 units of Taq DNA polymerase, and $250 \mathrm{nM}$ of each primer (rPLU5 and rPLU6). The PCR and nested PCR conditions were as follows: $95^{\circ} \mathrm{C}$ for 5 minutes; $94^{\circ} \mathrm{C}$ for one minute, $58^{\circ} \mathrm{C}$ for two minutes; extension at $72^{\circ} \mathrm{C}$ for two minutes; 25 cycle and final extension at $72^{\circ} \mathrm{C}$ for 7 minutes. About $2 \mu \mathrm{l}$ of the first amplification products were served as the DNA template for each of the $20 \mu$ of the second PCR amplification. The concentrations of the primers and other constituents were identical to the first amplification, except that 0.3 unit of Taq DNA polymerase was used. PCR products, after amplification were subjected to gel electrophoresis on a $1.5 \%$ agarose gel, stained with ethidium bromide, and visualized under UV light [44]. These reactions were then subjected to $1.5 \%$ agarose gel allowed to go through electrophoresis for 45 minutes. The gel-casting tray with the gel was then removed 
and placed in the gel-documentation machine connected to the computer system for imaging of parasite DNA bands [45].

\subsection{Statistical Analysis}

Data collected were analyzed statistically using SPSS version 19. The numbers of true positive (TP), true negatives (TN), false positive (FP) and false negatives $(\mathrm{FN})$ were used to calculate: Sensitivity $=\mathrm{TP} /(\mathrm{TP}+\mathrm{FN}) \times 100$; Specificity $=$ $\mathrm{TN} /(\mathrm{TN}+\mathrm{FP}) \times 100$; The Positive Predictive Value $(\mathrm{PPV})=\mathrm{TP} /(\mathrm{TP}+\mathrm{FP}) \times$ 100; The Negative Predictive Value $(\mathrm{NPV})=\mathrm{TN} /(\mathrm{FN}+\mathrm{TN}) \times 100$ and Diagnostic Accuracy $(D A)=T P+T N / T o t a l$ No. of patients $\times 100$. Analysis of variance was done on the levels of parasitaemia.

\section{Results}

\subsection{Results of Blood Microscopy}

Hundred (100) blood samples were collected for microscopy from patients attending Plateau Specialist Hospital, Jos and the University of Jos Health Clinic. The slides (Thin and Thick Films) for each of the hundred (100) blood samples were prepared, examined, and screened for the species of Plasmodium-Plasmodium falciparum, Plasmodium vivax, Plasmodium ovale, and Plasmodium malariae, using the $\times 10, \times 40$, and $\times 100$ objectives respectively of the light microscope. Out of the hundred (100) blood samples collected, thirty (30\%) were positive while seventy (70\%) were negative (Figure 2). The Parasite Density Per $\mu \mathrm{l}$ of blood of $30(+)$ positive samples per 100 HPF were then categorized and placed appropriately as shown on the graphical representation of the malaria parasitaemia and various levels of parasitaemia in patients in Figure 3. The Analysis of variance (ANOVA: Single Factor) was done for the statistical analysis of the data using the SPSS (Table 1).

Table 1. ANOVA: Single factor.

\begin{tabular}{|c|c|c|c|c|c|c|}
\hline \multicolumn{7}{|c|}{ SUMMARY } \\
\hline Groups & Count & Sum & Average & Variance & & \\
\hline Column 1 & 12 & 976 & 81.33333 & 2208.97 & & \\
\hline Column 2 & 12 & 4496 & 374.6667 & 120108.6 & & \\
\hline Column 3 & 12 & 48,560 & 4046.667 & $20,365,333$ & & \\
\hline Column 4 & 12 & 52,520 & 4376.667 & $41,896,861$ & & \\
\hline \multicolumn{7}{|l|}{ ANOVA } \\
\hline Source of Variation & SS & df & MS & $\mathrm{F}$ & $\mathrm{P}$-value & F crit \\
\hline Between Groups & $1.92 \mathrm{E}+08$ & 3 & $63,868,289$ & 4.095138 & 0.011976 & 2.816466 \\
\hline Within Groups & $6.86 \mathrm{E}+08$ & 44 & $15,596,128$ & & & \\
\hline Total & $8.78 \mathrm{E}+08$ & 47 & & & & \\
\hline
\end{tabular}




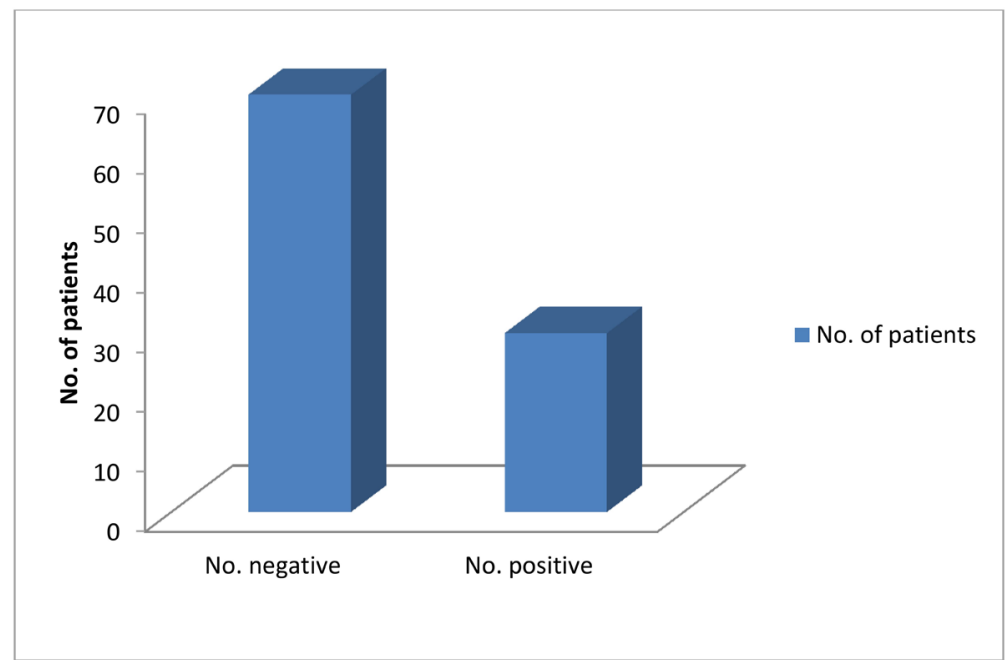

Figure 2. Categorizations of patients.

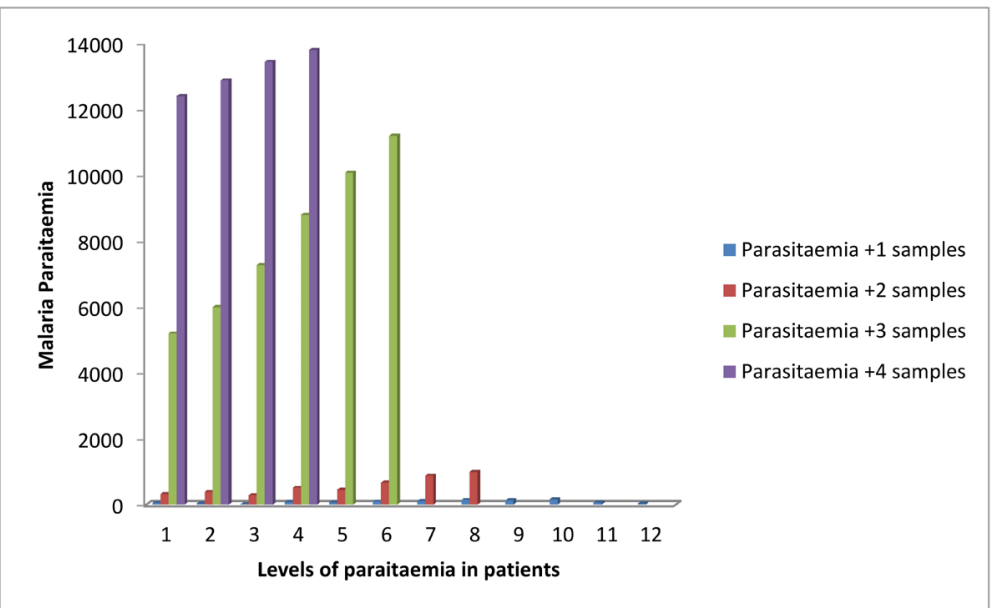

Figure 3. Malaria parasitaemia (parasites density per $\mu$ l of blood of $30(+)$ positive samples per $100 \mathrm{HPF}$ ) and various levels of parasitaemia in patients.

From the above table, the variance ratio (0.011976) calculated is less than F-critical (2.816466) at 5\% (0.05). This implies that there is no significant difference in the parasitaemia levels of the four groups/categories of patients.

Correlation was done based on the parasitaemia levels and the patients through the following graphical representations (Figures 4-7). The correlatation of determination $\left(\mathrm{R}^{2}\right)$ for Figures 4-7 indicates that all the parasitaemia levels have the ratio of above eighty five percent (85\%) explained variation to the total variation at each level of parasitaemia, with the +3 and +4 having above ninety nine percent (99\%).

\subsection{Results of RDTS}

For confirmation, the thirty blood samples that tested positive by microscopy were also tested using RDT kits (RDT Cassettes) which included optimal-IT ${ }^{\circ}$ and one-step RDT. 


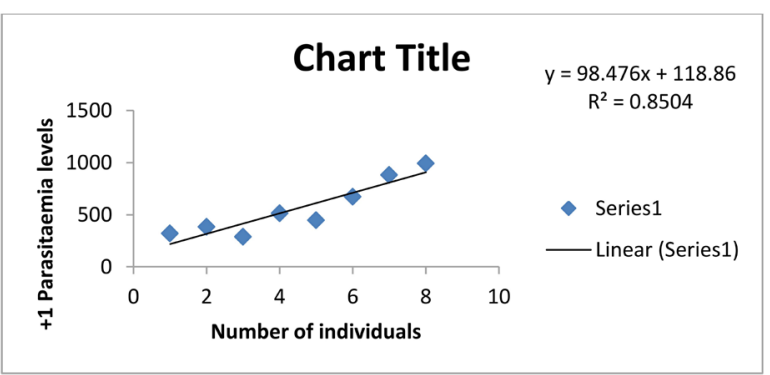

Figure 4. Correlation of $1+$ parasitaemia and the number of individual.

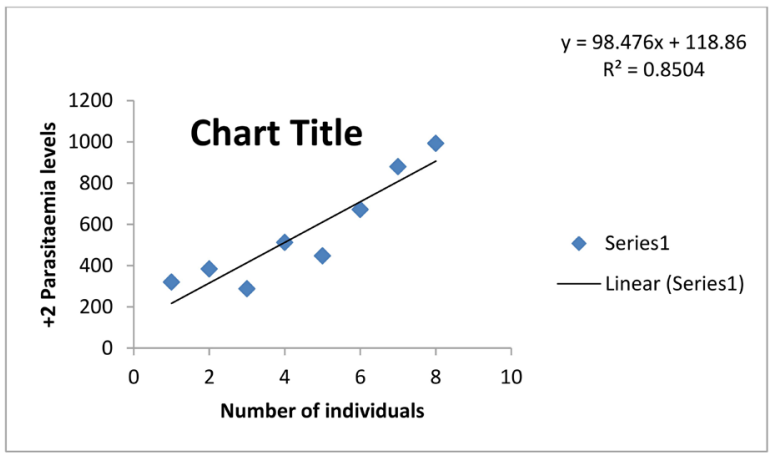

Figure 5. Correlation of $2+$ parasitaemia and the number of individual.

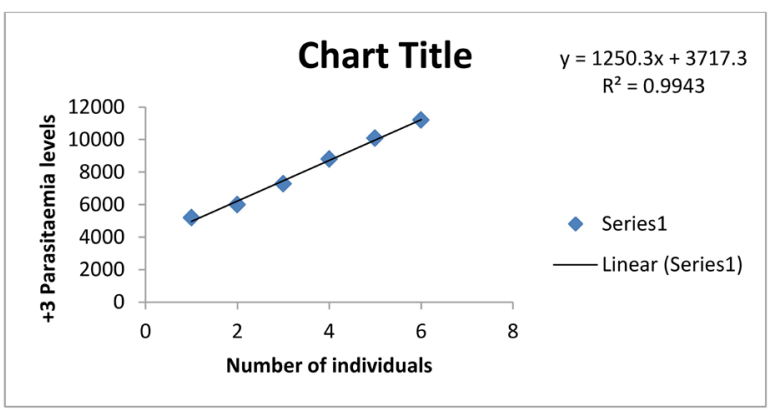

Figure 6. Correlation of $3+$ parasitaemia and the number of individuals.

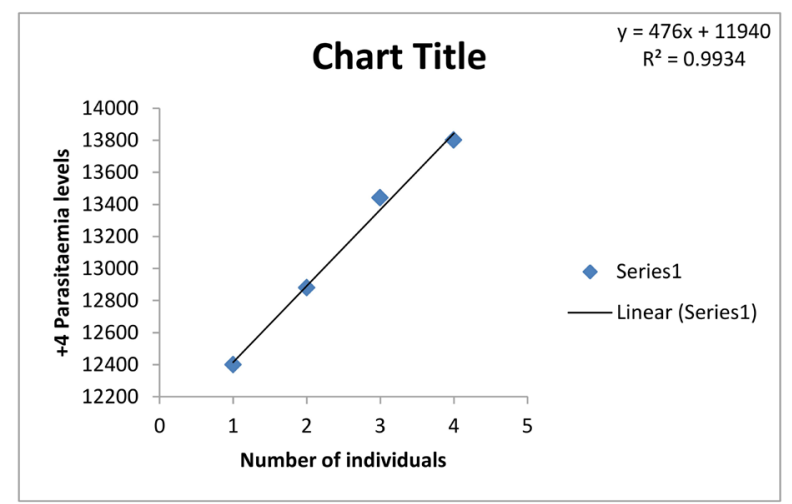

Figure 7. Correlation of $4+$ parasitaemia and the number of individuals. 


\subsubsection{Results of Optimal-IT ${ }^{\circledR}$}

Out of the thirty (30) blood samples tested, eighteen (60\%) tested positive while twelve (40\%) tested negative by the optimal IT ${ }^{\circledast}$ RDT. The following plates show the images of the results of the optimal-IT ${ }^{\infty}$

\subsubsection{Results for One-Step RDTs}

Out of the thirty (30) blood samples tested, eighteen (60\%) tested positive while twelve (40\%) tested negative by the one step RDTs.

\subsection{Results of DNA Extraction}

Ten out of the eighteen sensitive RDTs' positive samples were taken for molecular assay at the Applied molecular Biology Laboratory of Applied Biotechnology Division-National Veterinary Research Institute Vom, Plateau State, Nigeria. An almost equal number were selected from each of the various categories of parasitaemia levels. The DNAs of the parasites (Plasmodium species) were extracted from the positive blood samples using the Blood DNA Mini Extraction Kit. The DNA templates of the parasites in each of the blood samples were filtered out after the process of extraction. The following plates show the DNA extraction process.

\subsubsection{Results of PCR}

The parasites DNA templates extracted were used to run the PCR (Polymerase Chain Reaction). The parasite DNA templates for each sample were amplified. The PCR products (amplicons) were used to run the electrophoresis.

Agarose gel Electrophoresis: Agarose gel electrophoresis was performed on the amplicons using $100 \mathrm{bp}$ DNA Ladder (Molecular Marker) respectively. Clear bands were produced for the amplicons (PCR products). The following plate shows the bands as visualized under the ultra violet Trans illuminated machines. The first two lines (L1 - 2) were for 4+; lines 3 - 4 for $3+$; lines 5 - 7 for 2+; lines 8 - 9 for 1+; while line 10 for the negative control. In all the ones that produced bands on the agarose gel, a $250 \mathrm{bp}$ was recorded. The results from the bands also showed that amplifications of the bands from $4+, 3+, 2+$ were almost all positive, whereas those of $1+$ were negative (Figure 8).

\subsubsection{Results of NESTED PCR}

The PCR products after the first amplification reaction were allowed to run for the second reaction (Nested PCR) for the detection of the Plasmodium species. The nPCR products (amplicons) were then used to run the electrophoresis.

Agarose gel Electrophoresis: Agarose gel electrophoresis was performed on the amplicons using 100 bp DNA Ladder (Molecular Marker) respectively. Clear bands were produced for the amplicons (nPCR products). The following plate shows the bands as visualized under the ultra violet Trans illuminated machine. The top wells were for the Plasmodium falciparum, while the bottom wells were for Plasmodium vivax. The bands confirm the amplifications for Plasmodium falciparum with $250 \mathrm{bp}$ recorded, whereas the results for the Plasmodium vivax 
were negative (Figure 9).

The summary of the effectiveness of the various diagnostic methods is as shown in Figure 10.

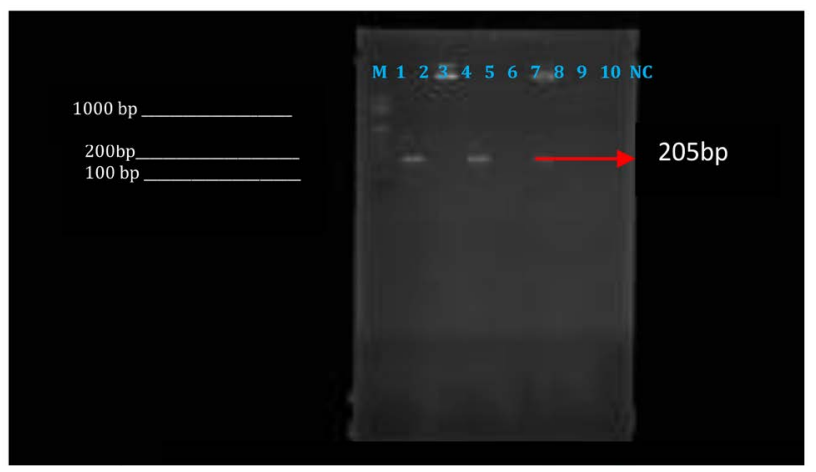

Figure 8. Showing electrophoresis result of PCR of the ten (10) samples using rplu5 and rplu5 with 100 bp DNA ladder. Negative control (NC) was used.

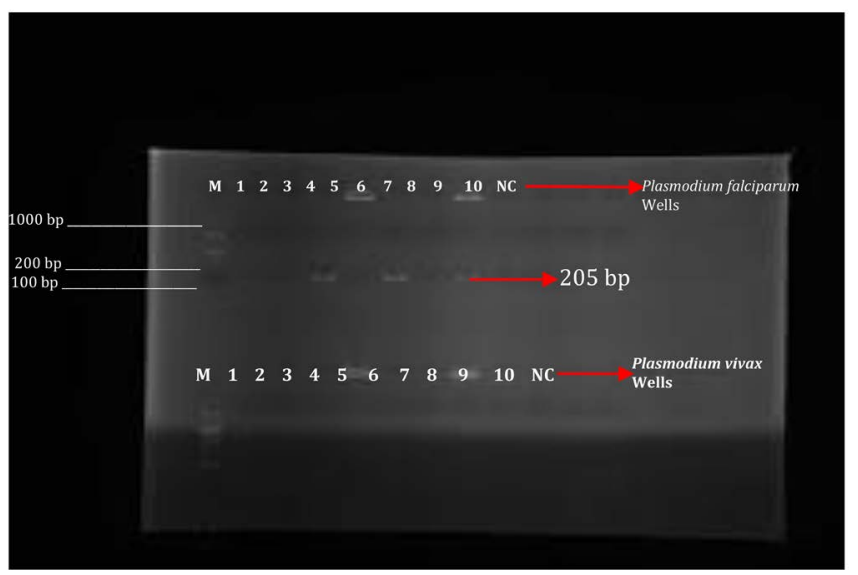

Figure 9. Showing electrophoresis result of $\mathrm{nPCR}$ of the ten (10) samples using rFAL1 and rFAL2, and rVIV1 and rVIV2 with 100 bp DNA ladder. Three samples showed positive for Plasmodium falciparum while none was positive for $P$. vivax.

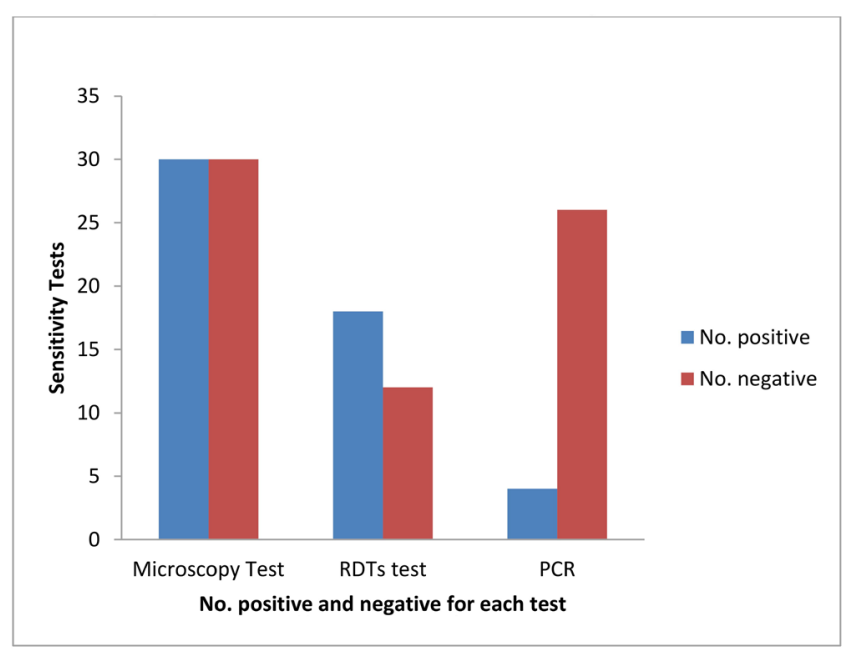

Figure 10. Effectiveness of the various sensitivity tests. 


\section{Discussion}

In malaria endemic regions where the pyretic illness can become rapidly fatal, an encompassing high sensitive diagnostic methods with precisions of higher specificity are important in order to reduce unnecessary treatment with antimalarial drugs and to improve on accurate diagnosis of other pyretic illnesses. The results obtained from the study clearly indicates that there were high chances that presumptive diagnosis of people could be higher when compared with conventional malaria diagnostic methods, implying that there could be high chances of treating who are actually not sick of malaria, a false-positive phenomenon. This agrees with the reports of WHO [46] [47], that presumptive treatments based on axillary temperature continue to remain a great challenge and therefore recommend that confirmation of parasites in body fluids for all suspected cases and treatments should only be applied to clinical feeling when parasitological diagnosis that requires a sensitivity of more than $90 \%$ are not available.

The results from the microscopy indicates that out of the 100 blood samples collected and tested, 30 (30\%) were positive to microscopy while 70 (70\%) were negative to microscopy. Moreover, only Plasmodium falciparum (with predominantly the lowest parasitaemia level of $1+$ ) were seen in all the 30 positive samples. $P$. vivax, $P$. ovale, $P$. malariae, and $P$. knowlesi, were not seen. This conforms to the report by Duffy et al. [3] [48] [49] where evidence has it that Plasmodium falciparum is majorly recorded among Africans who are Duffy-negative, discourages $P$. vivax that is Duffy-positive but encourages $P$. ovale which is similar to $P$. vivax to infect Duffy-negative persons. Although the hypnozoites $P$. vivax or $P$. ovale, nor the Ziemann's dot $P$. malariae were not seen in our study as reported by Goselle et al. [50] from Nigeria and Moyeh et al. [51] from Cameroun; it is worthy to note that as reported by Miller et al. [52] [53], that a mutation that abolishes the expression of the Duffy antigen receptor of chemokines on the surface of red blood cells (the so-called "Duffy-negative phenotype") approaches fixation in Western and Central Africa, and confers almost complete protection from $P$. vivax parasitaemia could still be at play.

RDT was performed as a confirmatory test to all the 30 samples that tested positive to microscopy (gold standard), out of which 18 tested positive to both the one-step RDT and the OPTIMAL-IT RDT. Both the one-step RDT and the optimal-IT ${ }^{\oplus}$ RDT were positive to only Plasmodium falciparum and negative to other Plasmodium species. The 18 samples that were recorded as $2+, 3+$, and $4+$ levels of parasitaemia, in the microscopy (gold standard), appeared positive in the RDT while the 12 samples recorded as $1+$ appeared negative. This has shown that at very low level of parasitaemia (1+), the RDT has a very low sensitivity, thus making it less reliable compared to the microscopy (gold standard). This agrees with the report by Wongsrichanalai et al. [54] which states that roughly, RDT sensitivity declines at parasite densities $<500 / \mu /$ blood for $P$. falciparum and $<5000 / \mu /$ blood for $P$. vivax. In comparison with the light microscopy test, it was observed that there was a significant difference $(\mathrm{P}<0.05)$. This disagrees with 
the findings of Moyeh et al. [51], who although used a SD Bioline RDT (a much higher sensitivity diagnostic test) discovered in comparison with microscopy that there was no significant difference between the two diagnostic methods. In spite essential limitations encountered in using light microscopy [55], the diagnostic sensitivity was still high when compared to that of OPTIMAL-IT* RDT.

Molecular assay indicates that out of the eighteen (18) RDTs' positive samples, ten (10) samples were randomly selected from each of the different levels of parasitaemia to run PCR and nested PCR. The PCR results indicated that three 3 (30\%) out of the $10(100 \%)$ selected positive samples bands were positive by PCR to Plasmodium and were successfully amplified from parasitaemia levels 4+; 3+ and $2+$, whereas negative for $1+$. While 7 (70\%) were negative to PCR. The 7 negative samples by PCR which were earlier on reported positive by microscopy could be as a result of false positive microscopy results. False positive microscopy results of some samples might have been reported as positive perhaps due to poor blood film preparation which might have generated artifacts mistaken for malaria parasites. This correlates to the report by Wongsrichanalai et al. [54] that poor blood film preparation generates artifacts commonly mistaken for malaria parasites, including bacteria, fungi, stain precipitation, and cell debris. In addition, the false negative results produced by OPTIMAL-IT* RDT could also be attributed to either be due to variation in field isolates from intraspecies HRP2/HRP3 sequence [56], or due to deletions, or mutations of HRP2/HRP3 where the parasites no longer produces the antigen or produces a mutant antigen not recognized by antibodies on the test strip [57] [58]. A further confirmation as elucidated by Moyeh et al. [51] in comparing microscopy and SD Bionline RDT, is that taking of antimalarial drugs could affect sensitivity of a test. That although these samples may not have intact parasites, but the gene product of HRP2 gene may still be in circulation and as noted by Sema et al. [59] and Kain et al. [60] that at times as long as 31 days after treatment. PCR identified three samples from the ten initially confirmed by both microscopy and RDTs'. The negative samples obtained from PCR could be due to the non-target Plasmodium species gene fragment recognized by the oligonucleotide primers which could have been deleted or mutated [57]. Instructively, as observed in endemic areas by Sema et al. [59], that there may be no correlation between parasitaemia and antigenemia due to the early clearance of parasites by the immune system in an infection thus, leading to false positive RDT results or alternatively they could have been sequestered in deep capillaries of certain organs like the spleen, liver or bone marrow. Consequently, such false positive RDT samples will be negative by PCR due to the presence of circulating antigens in blood samples.

Nested PCR was done on the ten (10) selected samples to detect the species of malaria parasite present. The nested PCR was able to detect only Plasmodium falciparum infection in the three (3) samples that appeared positive by the PCR. This agrees with the findings of Moyeh et al. [51]; Bigoga et al. [61]; Goselle et al. [62] who all reported an economically viable number of Plasmodium falciparum 
when compared to Plasmodim ovale in Coastal Cameroun; Tiko and Plasmodium malariae in Nigeria respectively. But this is at variance with the findings of Fru-Cho et al. [63] who reported substantial number of Plasmodium vivax in Coastal Cameroun and more number of Plasmodium malariae compared to Plasmodium ovale by Achonduh et al. [64] in Bangolan. The non-presence of other species in our results does not negate their presence. This could be probably due to the area of study. Evidence has remain a difficult task that Plasmodium falciparum is majorly recorded among Africans who are Duffy-negative, Discourages Plasmodium vivax that is Duffy-positive but encourages Plasmodium ovale which is similar to Plasmodium vivax to infect Duffy negative persons [3] [49]. Consequently, according to the WHO report [4] for 2015-2016 in Nigeria, malaria report indicated that on both admissions and deaths due to Plasmodium vivax which was hitherto not available between the years 2005-2011 suddenly emerges between the years 2012-2015 with a figure of about 10,000. The question begging for answers are: has the immune system of the duffy negative people become compromised or that Plasmodium vivax has developed certain features that enables it to adapt to the same pattern of infection like Plasmodium falciparum, or have certain characteristics like $P$. ovale or better still, that a case of highly imported Plasmodium vivax is being experienced and recorded?

\section{Conclusion}

This study which compared the four different diagnostic methods of diagnosing malaria parasites revealed the advantage of the microscopy (gold standard) in recording of the highest number of positive results compared to the RDT and the PCR. However, the negative results revealed by the RDT and subsequently by PCR could be as a result of the inability of the RDT and PCR to detect very low level of parasitaemia or false positive results revealed by the microscopy. Whereas it has been advocated by other researchers that certain diagnostic test with high diagnostic sensitivity like the SD Bionline could replace microscopy in remote areas with difficult access to reagents and electricity, our findings indicate the usage of SD Bionline alone could overlook certain levels of parasitaemia from a single blood film which could have been detected at early stage of infection,

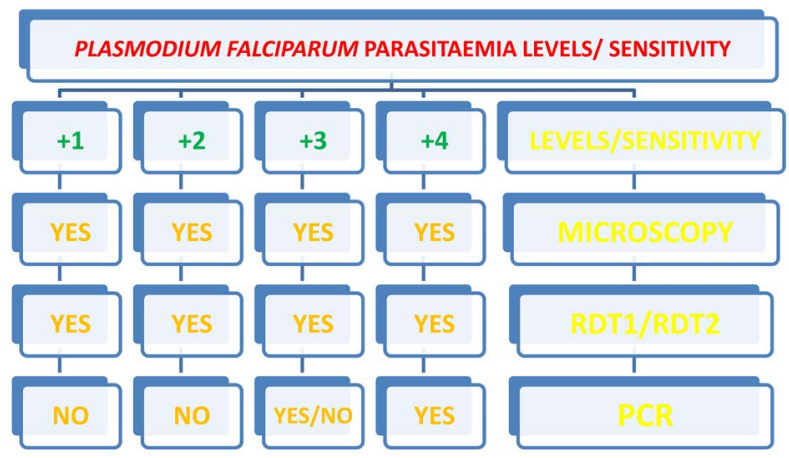

Figure 11. Summary of schematic representation of the results for the three sensitivity tests. 
thus complicating the modeling. Although our study in conclusion highlights the inadequacy of presumptive diagnosis to be far lower to the references for poised diagnosis of malaria. We therefore recommend that adequate efforts should be put in place to replace presumptive diagnosis practices with parasitological validation of the parasites before administration of ACT therapy. However, only Plasmodium falciparum was detected by all the four diagnostic methods (Figure 11).

\section{Recommendations}

While the use of microscopy method (gold standard) for diagnosing malaria parasites is recommended due to its low cost, its ability to reveal the morphology of malaria parasites and useful in the estimation of parasite density. The use of RDTs (are simple, quick to use, and interpret, and are potential alternatives to microscopy) are highly recommended for places where the facilities for microscopy are poor such as the rural areas. The PCR (Molecular assay) on the other hand is expensive and requires a very standard laboratory with well trained personnel. Therefore, it is recommended that Government and Non-Governmental Organization (NGOs) should invest in the provision of standard molecular laboratories and personnel training in our private and Government-owned hospitals, educational and research institutes of learning in poor resourced settings. This is necessary because PCR is one of the most modern methods of diagnosing malaria parasites to species and subspecies level, and has the capacity to detect very low levels of parasitaemia.

\section{Authors' Contributions}

Goselle O. Nanjul initiated, designed, supervised the studies and wrote the manuscript. Ajiji G. Yandu participated in sample collection, data processing, performed molecular analysis, and drafted the manuscript. Jambol Anvou, Sunday, T. Joseph, Idoko, Sunday, Udoh, S. Shedrach., Ejete, O. Charles and Ahmadu, M. Yahaya all participated in the molecular analysis and editing of the final manuscript. Awobode, O. Henrietta, Imandeh, N. Godwin, Matur, M. Bernard were involved in data entry and analysis, and supervised sample collection, data processing, and revision of the final manuscript. All authors approved the final version of the manuscript.

\section{Acknowledgements}

The authors are thankful to the administration of the Plateau Specialist Hospital, Jos who made available their facility for them to carry out this research work. They are also grateful to the participants who gave their consent to take part in the study. The study was a self-sponsored research work aiming at contributing to the wealth of malaria knowledge.

\section{Conflicts of Interest}

The authors declare that they have no conflicts of interest. 


\section{References}

[1] Tangpukdee, N., Duangdec, C., Wilairatana, P. and Krudsood, S. (2009) Malaria Diagnosis. The Korean Journal of Parasitol, 47, 93-102. https://doi.org/10.3347/kjp.2009.47.2.93

[2] WHO (2015) How Malaria Rapid Diagnostic Tests, (RDTs) Work.

[3] Goselle, O.N., Ajayi, O.O., Wuyep, A.P. and Mafuyai, H.B. (2016) Understanding the Gavssian Polygenic Traits Cum Latency and Reservoir: Climateric for Plasmodium Falciparun. Keystone Conference on Drug Discovery for Parasitic Disease (AS), Granlibakken, Abstract of Poster 3039.

[4] (2017) World Malaria Report. World Health Organization, Geneva. http://apps.who.int/iris

[5] Dawaki, S., Al-Mekhlafi, H.M., Ithoi, Ibrahim, J., Atroosh, W.M., Abdulsalam, A.M., Sady, H., Elyana, F.N., Adamu, A.U., Yelwa, S.I, Ahmed, A. and Lau, Y.L. (2016) Is Nigeria Winning the Battle Against Malaria? Prevalence, Risk Factors and KAP Assessment among Hausa Communities in Kano State. Malaria Journal, 15, 351. https://doi.org/10.1186/s12936-016-1394-3

[6] Roll Back Malaria Funding Gap Analysis (2017). https://rollbackmalaria.com/news/gap-analysis-shows-us10-billion-is-required-by-2 020-to-fully-implement-national-malaria-plans-in-35-countries

[7] Lubanga, R.G.N., Norman, S., Ewbank, D. and Karamagi, C. (1997) Maternal Diagnosis and Treatment of Children's Fever in an Endemic Malaria Zone of Uganda: Implications for the Malaria Control Programme. Acta Tropica, 68, 53-64. https://doi.org/10.1016/S0001-706X(97)00071-5

[8] Warhurst, D.C. and Williams, J.E. (1996) Laboratory Diagnosis of Malaria. Journal of Clinical Pathology, 49, 533-538. https://doi.org/10.1136/jcp.49.7.533

[9] Muhindo, H.M., Ilombe, G., Meya, R., Mitashi, P.M., Kutekemeni, A. and Gasigwa, D. (2012) Accuracy of Malaria Rapid Diagnosis Test Optimal-IT (R) in Kinshasa, the Democratic Republic of Congo. Malar Journal, 11, 224-226. https://doi.org/10.1186/1475-2875-11-224

[10] Kilian, H., Metzger, J., Mutschelknauss, G., Kabagambe, P., Langi, R., Korte and Sonnenburg, V. (2000) Reliability of Malaria Microscopy in Epidemiological Studies: Results of Quality Control. Tropical Medicine and International Health, 5, 3-8. https://doi.org/10.1046/j.1365-3156.2000.00509.x

[11] Garcia, A., Kirimoama, S., Marlborough, D., Leafasia, J. and Rieckmann, K.H. (1996) Immunochromatographic Test for Malaria Diagnosis. The Lancet, 347, 1549. https://doi.org/10.1016/S0140-6736(96)90700-X

[12] Craig, M.H. and Sharp, B.L. (1997) Comparative Evaluation of Four Techniques for the Diagnosis of P. falciparum Infections. Transaction of the Royal Society of Tropical Medicine and Hygiene, 91, 279-282. https://doi.org/10.1016/S0035-9203(97)90074-2

[13] Srinivasan, S., Moody, A.H. and Chiodini, P.L. (2000) Comparison of Blood-Film Microscopy, the OptiMAL Dipstick, Rhodamine-123 Fluorescence Staining and PCR, for Monitoring Antimalarial Treatment. Annals Tropical Medicine and Parasitology, 94, 227-232. https://doi.org/10.1080/00034983.2000.11813533

[14] Murray, C.K., Bell, D., Gasser, R.A. and Wongsrichanalai, C. (2003) Rapid Diagnostic Testing for Malaria. Tropical Medicine and International Health, 8, 876-883. https://doi.org/10.1046/j.1365-3156.2003.01115.x

[15] Kakkilaya, B.S. (2003) Rapid Diagnosis of Malaria. Laboratory Medicine, 8, 602-608. 
https://doi.org/10.1309/J4ANKCCJ147JB2FR

[16] Kyabayinze, D.J., Tibenderana, J.K., Odong, G.W., Rwakimari, J.B. and Counihan, H. (2008) Operational Accuracy and Comparative Persistent Anti-Genicity of HRP2 Rapid Diagnostic Tests for Plasmodium falciparum Malaria in a Hyperendemic Region of Uganda. Malar Journal, 7, 221. https://doi.org/10.1186/1475-2875-7-221

[17] Endeshaw, T., Gebre, T., Ngondi, J., Graves, P.M., Shargie, E.B., Ejigsemahu, Y., Ayele, B., Yohannes, G., Teferi, T., Messele, A., et al. (2008) Evaluation of Light Microscopy and Rapid Diagnostic Test for the Detection of Malaria under Operational Field Conditions: A Household Survey in Ethiopia. Malaria Journal, 7, 118-120. https://doi.org/10.1186/1475-2875-7-118

[18] Ratsimbasoa, A., Fanazava, L., Radrianjafy, R., Ramilijaona, J., Rafano-Mezantsoa, H. and Menard, D. (2008) Evaluation of Two New Immunochromatographic Assays for Diagnosis of Malaria. American Journal of Tropical Medicine and Hygiene, 79, 670-672. https://doi.org/10.4269/ajtmh.2008.79.670

[19] Lee, S.W., Jeon, K., Jeon, B.R. and Park, I. (2008) Rapid Diagnosis of Vivax Malaria by the SD Bioline Malaria Antigen Test When Thrombocytopenia Is Present. Journal of Clinical Microbiology, 46, 939-942. https://doi.org/10.1128/JCM.02110-07

[20] Kim, S.H., Nam, M.H., Roh, K.H., Park, H.C., Nam, D.H., Park, G.H., Han, E.T., Klein, T.A. and Lim, C.S. (2008) Evaluation of a Rapid Diagnostic Test Specific for Plasmodium vivax. Tropical Medicine and International Health, 13, 1495-1500. https://doi.org/10.1111/j.1365-3156.2008.02163.x

[21] WHO (2004) The Use of Malaria Diagnostic Tests. WHO Regional Office for the Western Pacific (WPRO), Manila.

[22] Moody, A. (2002) Rapid Diagnostic Tests for Malaria Parasites. Clinical Microbiology Reviews, 15, 66-78. https://doi.org/10.1128/CMR.15.1.66-78.2002

[23] Zakeri, S., Kakar, Q., Ghasemi, F., et al. (2010) Detection of Mixed Plasmodium falciparum \& P. vivax Infections by Nested-PCR in Pakistan, Iran \& Afghanistan. Indian Journal of Medical Research, 132, 31-35.

[24] Assmar, M., Terhovanessian, A., Jahani, M.R., et al. (2003) Molecular Epidemiology of Malaria in Endemic Areas of Iran. The Southeast Asian Journal of Tropical Medicine and Public Health, 34, 15-19.

[25] Ebrahimzadeh, A., Polshakan, M., Qureshi, M.I. and Sharifi, I. (2006) Reliable DNA Extraction on Historical Malaria Negative Smears Conducted to Nested PCR in South-East of Iran. Biotechnology, 5, 353-357. https://doi.org/10.3923/biotech.2006.353.357

[26] Ebrahimzadeh, A., Fouladi, B. and Fazaeli, A. (2007) High Rate of Detection of Mixed Infections of Plasmodium vivax and Plasmodium falciparum in South-East of Iran, Using Nested PCR. Parasitology International, 56, 61-64. https://doi.org/10.1016/j.parint.2006.12.001

[27] Snounou, G., Viriyakosol, S., Jarra, W., Thaithong, S. and Brown, K.N. (1993) Identification of the Four Human Malaria Parasite Species in Field Samples by the Polymerase Chain Reaction and Detection of a High Prevalence of Mixed Infections. Molecular and Biochemical Parasitology, 58, 283-292. https://doi.org/10.1016/0166-6851(93)90050-8

[28] Oriero, F.C., Jacobs, J., Geetruyden, J.V., Nwakanma, D. and D’Alessandra, U. (2015) Molecular Based Isothermal Tests for Field Diagnosis of Malaria and Their Potential Contribution to Malaria Elimination. Journal of Anti-Microbial Chemotherapy, 70, 2-13. https://doi.org/10.1093/jac/dku343

[29] Zakeri, S., Najafabadi, S.T., Zare, A. and Djadid, N.D. (2002) Detection of Malaria 
Parasites by Nested PCR in South-Eastern, Iran: Evidence of Highly Mixed Infections in Chahbahar District. Malaria Journal, 8, Article 2. https://doi.org/10.1186/1475-2875-1-2

[30] Zakeri, S., Mamaghani, S., Mehrizi, A.A., et al. (2004) Molecular Evidence of Mixed $P$. vivax and $P$. falciparum Infections in Northern Islamic Republic of Iran. Eastern Mediterranean Health Journal, 10, 336-342.

[31] Google Wikipedia (2015) Nested Polymerase Chain Reaction. https://en.wikipedia.org

[32] Epidi, T.T., Nwani, C.D. and Ugorji, N.P. (2008) Prevalence of Malaria in Blood Donors in Abakaliki Metropolis, Nigeria. Scientific Research and Essay, 3, 162-164.

[33] Microbe Online (2010) Thick and Thin Blood Smear for Malaria Diagnosis.

[34] World Health Organization (2000) New Perspectives in Malaria Diagnosis. World Health Organization, Geneva.

[35] World Health Organization (1991) Basic Malaria Microscopy: Part I Learner's Guide; Part II Tutor's Guide. Tech. Rep., WHO, Geneva.

[36] Alkhiary, W. (2015) Evaluation of the Diagnostic Performance of Optimal IT $^{\circ}$ Test for the Detection of Plasmodium falciparum in South-West Saudi Arabia. Journal of Blood Disorders and Transfusion, 6, 272. https://doi.org/10.4172/2155-9864.1000272

[37] World Health Organization (2018) Rapid Diagnostic Tests.

[38] Panigrahi, B.K., Kerketta, A.S., Mohapatra, A., et al. (2011) Effect of Construction of an Irrigation Canal on Malaria Situation in Two Primary Health Centres of Dhenkanal District of Orissa, India. Tropical Biomedicine, 28, 76-84.

[39] Incardona, S., Chy, S. and Chivetal, L. (2005) Large Sequence Heterogeneity of the Small Subunit Ribosomal RNA Gene of Plasmodium ovale in Cambodia. American Journal of Tropical Medicine and Hygiene, 72, 719-724.

https://doi.org/10.4269/ajtmh.2005.72.719

[40] FGRS (2011) Protocol: Re-Suspending PCR Primers.

[41] Stupar Lab (1991) Primers. University of Minnesota.

[42] Quick gDNA Miniprep, the Epigenetic Company (2018) DNA Extraction Procedure Manual.

[43] Wenger, E.A. and Eckhoff, P.A. (2013) A Mathematical Model of the Impact of Present and Future Malaria Vaccines. Malaria Journal, 12, 10. https://doi.org/10.1186/1475-2875-12-126

[44] Mohammazadeh, T., Hatam, G., Kalantari, M., Sarkari, B., Motazedian, M.H., Sadjjadi, S.M. and Safari, R. (2014) Molecular and Microscopic-Based Characterization of Plasmodium spp. in Fars and Hormozgan Provinces, South of Iran. Journal of Tropical Medicine, 2, 1-6. https://doi.org/10.1155/2014/935469

[45] Biotechnology Division, Electrophoresis Lab., NVRI Vom (2018) Agarose Gel Preparation and Electrophoresis Procedure.

[46] World Health Organization (2010) Global Report on Antimalarial Drug Efficacy and Drug Resistance: 2000-2010. Tech. Rep., World Health Organization.

[47] World Health Organisation RS/2003/GE/05 (PHL) (2003) Malaria Rapid Diagnosis: Making It Work. Informal Consultation on Field Trials and Quality Assurance on Malaria Rapid Diagnostic Tests. Tech. Rep., WHO Regional Office for the Western Pacific.

[48] Duffy, P.E., Sahu, T., Akue, A., Milman, N. and Anderson, C. (2012) Pre-Erythrocytic Malaria Vaccines: Identifying the Targets. Expert Review of Vaccines, 11, 1261-1280. 
https://doi.org/10.1586/erv.12.92

[49] Bogistch, B.J., Carter, C.E. and Oeltmann, T.N. (2012) Human Parasitology. Fourth Edition, Elsevier Academic Press, Amsterdam, 448.

[50] Goselle, O.N., Onwuliri, C.O.E. and Onwuliri, V.A. (2009) Malaria Infection in HIV/AIDS Patients and Its Correlation with Packed Cell Volume (PCV). Journal of Vector Borne Diseases, 46, 205-211.

[51] Moyeh, M.N., Ali, I.M., Njimoh, D.L., Nji, A.M., Netongo, P.M., Evehe, M.S., Atogho-Tiedeu, B., Ghogomu, S.M., Wilfred, F. and Mbacham, W.F. (2019) Comparison of the Accuracy of Four Malaria Diagnostic Methods in a High Transmission Setting in Coastal Cameroon. Journal of Parasitological Research, 2019, Article ID: 1417967. https://doi.org/10.1155/2019/1417967

[52] Miller, L.H., Mason, S.J., Clyde, D.F. and McGinniss, M.H. (1976) The Resistance Factor to Plasmodium vivax in Blacks. The Duffy-Blood-Group Genotype, FyFy. New England Journal of Medicine, 295, 302-304. https://doi.org/10.1056/NEJM197608052950602

[53] Howes, R.E., Patil, P.A., Piel, F.B., Nyangiri, A.O., Kabaria, W.C., Gething, P.W., Zimmerman, P.A., Barnadas, C., Beall,C.M., Gebremedhin, A., Ménard, D., Williams, T.N., Weatherall, D.J. and Hay, S.I. (2011) The Global Distribution of the Duffy Blood Group. Nature Communications, 2, 266.

http://www.nature.com/naturecommunications https://doi.org/10.1038/ncomms1265

[54] Wongsrichanalai, C., Barcus, M.J., Muth, S., Sutamihardja, A. and Wernsdorfer, W.H. (2007) A Review of Malaria Diagnostic Tools: Microscopy and Rapid Diagnostic Test (RDT). American Journal of Tropical Medicine and Hygiene, 77, 119-127. https://doi.org/10.4269/ajtmh.2007.77.119

[55] Mens, P., Spieker, N., Omar, S., Heijnen, M., Schallig, H. and Kager, P.A. (2007) Is Molecular Biology the Best Alternative for Diagnosis of Malaria to Microscopy? A Comparison between Microscopy, Antigen Detection and Molecular Tests in Rural Kenya and Urban Tanzania. Tropical Medicine and International Health, 12, 238-244.

[56] Lee, N., Baker, J., Andrews, K.T., et al. (2006) Effect of Sequence Variation in Plasmodium falciparum Histidine-Rich Protein 2 on Binding of Specific Monoclonal Antibodies: Implications for Rapid Diagnostic Tests for Malaria. Journal of Clinical Microbiology, 44, 2773-2778. https://doi.org/10.1128/JCM.02557-05

[57] Gamboa, D., Ho, M.F., Bendezu, J., et al. (2010) A Large Proportion of P. falciparum Isolates in the Amazon Region of Peru Lack pfhrp2 and pfhrp3: Implications for Malaria Rapid Diagnostic Tests. Public Library of Science One, 5, e8091. https://doi.org/10.1371/journal.pone.0008091

[58] Kumar, N., Pande, V., Bhatt, R.M., et al. (2013) Genetic Deletion of HRP2 and HRP3 in Indian Plasmodium falciparum Population and False Negative Malaria Rapid Diagnostic Test. Acta Tropica, 125, 119-121. https://doi.org/10.1016/j.actatropica.2012.09.015

[59] Sema, M., Alemu, A., Bayih, A.G., et al. (2015) Evaluation of Non-Instrumented Nucleic Acid Amplification by Loop-Mediated Isothermal Amplification (NINALAMP) for the Diagnosis of Malaria in Northwest Ethiopia. Malaria Journal, 14, 44. https://doi.org/10.1186/s12936-015-0559-9

[60] Kain, K.C., Kyle, D.E., Wongsrichanalai, C., et al. (1994) Qualitative and SemiQuantitative Polymerase Chain Reaction to Predict Plasmodium falciparum Treatment Failure. The Journal of Infectious Disease, 170, 1626-1630. 
https://doi.org/10.1093/infdis/170.6.1626

[61] Bigoga, J.D., Manga, L., Titanji, V.P.K., Coetzee, M. and Leke, R.G.F. (2007) Malaria Vectors and Transmission Dynamics in Coastal South-Western Cameroon. Malaria Journal, 6, 5. https://doi.org/10.1186/1475-2875-6-5

[62] Goselle, O.N., Onwuliri, C.O.E. and Onwuliri, V.A. (2007) Malaria and the Effect of Malaria Parasitaemia on Albumin Level among HIV/AIDS-Patients in Jos, Nigeria. Journal of Medical Science, 7, 1187-1191. https://doi.org/10.3923/jms.2007.1187.1191

[63] Fru-Cho, J., Bumah, V.V., Safeukui, I., Nkuo-Akenji, T., Titanji, V.P.K. and Haldar, K. (2014) Molecular Typing Reveals Substantial Plasmodium vivax Infection in Asymptomatic Adults in a Rural Area of Cameroon. Malaria Journal, 13, 170. https://doi.org/10.1186/1475-2875-13-170

[64] Achonduh, O.A., Ekollo, A.H., Atogho-Tiedeu, B., et al. (2013) Pre-Dominance of Plasmodium malariae-falciparum Co-Infection by Molecular Speciation in Bangolan, North West Region of Cameroon. Life Science Journal, 7, 599-606. https://doi.org/10.17265/1934-7391/2013.06.006 